\title{
Exact spectra, spin susceptibilities and order parameter of the quantum Heisenberg antiferromagnet on the triangular lattice.
}

\author{
B. Bernu* ${ }^{*}$ P. Lecheminant ${ }^{* \dagger}$, C. Lhuillier *, and L. Pierre ${ }^{\ddagger}$
}

(October 11, 2018)

\begin{abstract}
Exact spectra of periodic samples are computed up to $N=36$. Evidence of an extensive set of low lying levels, lower than the softest magnons, is exhibited. These low lying quantum states are degenerated in the thermodynamic limit; their symmetries and dynamics as well as their finite-size scaling are strong arguments in favor of Néel order. It is shown that the Néel order parameter agrees with first-order spin-wave calculations. A simple explanation of the low energy dynamics is given as well as the numerical determinations of the energies, order parameter and spin susceptibilities of the studied samples. It is shown how suitable boundary conditions, which do not frustrate Néel order, allow the study of samples with $N=3 p+1$ spins. A thorough study of these situations is done in parallel with the more conventional case $N=3 p$.
\end{abstract}

PACS numbers: $75.10 \mathrm{~J} ; 75.40 \mathrm{M}$

Typeset using REVTEX

\footnotetext{
*Laboratoire de Physique Théorique des Liquides, Université P. et M. Curie, boite 121, 4 Place Jussieu, 75252 Paris Cedex. URA 765 of CNRS

${ }^{\dagger}$ Groupe de Physique Statistique, Université de Cergy-Pontoise, 95806 Cergy-Pontoise Cedex.

‡U.F.R. SEGMI, Université Paris-X, Nanterre, 92001 Nanterre Cedex.
} 


\section{INTRODUCTION}

The nature of the thermodynamic ground-state of the spin-1/2 Heisenberg antiferromagnetic hamiltonian, in two space dimensions, is still an open question. There have been considerable amounts of theoretical or numerical works on Heisenberg antiferromagnets[1-45] but few exacts results. It is known that in one and two dimensions the system is disordered at $T \neq 0$ [1], and that the one-dimensional system does not exhibit Néel order even at $T=0$. The two-dimensional case is more controversial. There is a rather large consensus on the existence of Néel order at $T=0$ on the (unfrustrated) square lattice [2 12]. The situation is much more puzzling as regards the triangular lattice case. It was indeed the first system to be proposed by Anderson [13] and Fazekas [14 as a candidate for a spinliquid. On this lattice the "frustration" implies that the classical system is not very stable $\left(E_{c l}=<2 \mathbf{s}_{i} \cdot \mathbf{s}_{j}>=-1 / 4\right)$, and the spin-wave calculations predict an important reduction (by about one half) of the sublattice magnetization by quantum fluctuations [15 17]. Perturbation theory [18], series expansions [19] and high temperature calculations [20] have been developed which suggest that the spin-wave calculations possibly underestimate this renormalization. Many variational calculations have been done exhibiting either ordered [6.21] or disordered solutions [22, 24].

In the square lattice case, numerical methods (Q.M.C., Ulam's or Trotter-Suzuki meth-

ods) have brought very interesting indications on Néel order [5,8-11]. Unfortunately these methods which allow to handle large samples cannot be applied to the triangular case: they lay on a property of positivity of offdiagonal matrix elements which is violated in the triangular case; it is the well known sign problem which plagues many studies of strongly correlated fermions. Exact diagonalizations of the hamiltonian are thus the last resort to gather new information on these models. This approach has been developed by other authors [25 32]. Most of them conclude to the absence of Néel order for the triangular Heisenberg antiferromagnet (THA) at $T=0$. But two requirements are to be met to analyze the raw numerical data: a consistent finite-size scaling analysis and a quantum definition of observables. With these two constraints we will show in this paper that all the numerical data point to an ordered ground-state for the THA.

As the second and perhaps more important objective we want to illustrate the spectral 
properties of an ordered quantum antiferromagnet on finite lattices and how they embody the characteristics of the symmetry breaking state (parts of these results have already been published [33]).

In section II, we first show the numerical spectra of the THA on periodic samples with $\mathrm{N}=9$ up to $\mathrm{N}=36$ spins and we present the essential characteristics of these spectra. Their low lying levels form two families: the first one contains an extensive number of states called QDJS in ref [33], which collapse to the ground-state as $N^{-1}$, and are characterized by the spatial symmetries of the classical Néel ground-state. The second family collapse more slowly to the ground-state as $N^{-1 / 2}$ and forms the familiar one magnon excitations. Such a structure has been conjectured a long time ago by Anderson in his seminal paper on antiferromagnets [2], and the subject has already been studied in the square lattice case [7,8, 34 , 37.

Section III is devoted to the understanding of the QDJS of the triangular Heisenberg hamiltonian. We show how the hypothesis of a Néel order explains the number of levels appearing for each spin, their symmetries and their dynamics. The analysis of these spectra gives a first information on the spin susceptibilities $\chi_{\perp}$ and $\chi_{\|}$of the THA. In order to check our general ideas, we extend the analysis to samples with $N=3 p+1$ spins. Suitable boundary conditions allow us to study situations which do not frustrate Néel order. We compare all the results of energies and susceptibilities with the first-order spin-wave results.

In section IV, we analyze the static spin-spin structure function and the Néel order parameter. We show how diagonalization results sustain the hypothesis of Néel order and how the analysis of some previous authors was in error, leading to confusing conclusions.

We conclude by a brief discussion of the interest and limitations of this approach.

In the Appendices, we develop the group theoretical analysis of the THA and explain our numerical method: this is a technical but crucial point in order to obtain the needed information in a minimum computer time, within memory capacities of today computers. We had for example to make sure that the low lying levels of all the different irreducible representations order as they should in the framework of our hypothesis. This leads us to compute the whole spectrum of the samples up to $N=21$ and a large number of low lying levels in each irreducible representations for all sample sizes up to $N=27$. 


\section{SPECTRA OF PERIODIC SAMPLES: AN OVERVIEW}

All the results presented here have been obtained by diagonalization of the spin- $1 / 2$ Heisenberg hamiltonian of periodic samples on the triangular lattice ( [25] and Appendix A for more details). The Heisenberg hamiltonian reads:

$$
\mathcal{H}=\sum_{<i, j>} 2 \mathbf{s}_{i} \cdot \mathbf{s}_{j}
$$

where the sum runs over pairs of nearest neighbors and $\mathbf{s}_{i}, \mathbf{s}_{j}$ are the spin- $1 / 2$ operators on sites $i$ and $j$.

In this section, we are concerned with periodic samples with $N=3 p$. Such samples do not frustrate the classical Néel order. A classical Néel state on the triangular lattice has coplanar spins with a three-fold rotational symmetry defining three magnetic sublattices $(A, B, C)$ (see fig.11). On each sublattice, the spins are ferromagnetically aligned and the angles between the magnetizations of two sublattices are $\pm 2 \pi / 3$. The total spin of a triangular plaquette is zero and the rotations of the spins around an $A B C$ triangle could be clockwise or counterclockwise defining two opposite helicities. The point symmetry group of these classical solutions is $C_{3 v}$, and the translational symmetry group is that of a sublattice, say $A$. These classical solutions break the translation and spin rotation invariance of Eq.1 whereas the quantum eigenstates that we will now consider do not.

Using group theory, described in Appendix A, we have computed the complete spectrum of Eq.1 for $N=9,12,21$. The 5 lowest energies have been computed for $N=27$ in all the irreducible representations (IR), and the 3 lowest energies for $N=36$ in the homogeneous states $\mathbf{k}=\mathbf{0}$, invariant under rotation. The energy spectra are given in fig.2 and the lowest

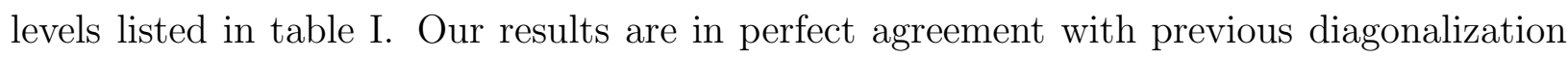
results 27,32 .

A first very striking feature of these spectra could be read in fig.2: the lowest energy levels in each spectra order with increasing $S$. This is strongly reminiscent of the theorem by Lieb and Mattis for bipartite lattices [38]. But there is presently no proof that the property holds for other lattices. We find this is true for other non bipartite problems like the Kagomé Heisenberg antiferromagnet or for the $J_{1}-J_{2}$ models on triangular and Kagomé lattices. 
The second striking feature of these spectra is the existence in each $S$ subspace of a family of low lying levels well separated from the others: we called these states the "QuasiDegenerate-Joint States" (QDJS) in ref. [33]. In fig.2, it is shown that the QDJS energies $\left(6 N<\mathbf{s}_{i} \cdot \mathbf{s}_{j}>\right)$ stand around a line $E=S(S+1) /\left(2 I_{N}\right)$, where the moment of inertia $I_{N}$ is essentially proportional to $N$ (see fig.3). This suggests that these levels collapse as $N^{-1}$ to the ground-state. We shall show in the next section that this family of $\mathcal{O}\left(N^{3}\right)$ QDJS has all the properties expected for the description of Néel quantum ground-states: in particular, the $C_{3 v}$ and magnetic sublattice translation invariance of the Néel states (they only contain the $\mathbf{k}=\mathbf{0}$ or $\pm \mathbf{k}_{0}$ IRs of the lattice translation operators where $\pm \mathbf{k}_{0}$ are the two wavevectors of the corners of the crystal Brillouin zone, mapping on the center of the magnetic Brillouin zone). The symmetry breaking Néel states are linear combinations of these QDJS.

To study higher excited states, let us first come back to the $N=9$ spectrum of fig. 1 . Above the basal line of QDJS one sees very clearly two families of levels.

The first excited-state family (horizontal bars in fig.(1) consists of eigenstates of the translation operator with $\mathbf{k} \neq \mathbf{0}$ and $\mathbf{k} \neq \pm \mathbf{k}_{0}$, they are typically states involved in one magnon excitations. What is usually called a magnon in solid state physics is in fact a spatial modulation of the Néel state. As the Néel states should be seen as linear combinations of the QDJS, the one magnon excitations are in fact linear superpositions of this first family of excited states. A one magnon excitation is thus pictured in the spectrum of fig.t as a $\Delta S=1$ collective excitation of the QDJS (three legs symbols family) towards the $k$ family of levels (this picture has been ascertained by the computation of the dynamic structure factor of the first QDJS of the $N=12$ sample [39]).

The second family of excited states (black triangles) belongs to the non trivial IRs of $C_{3}$ $\left[\mathcal{R}_{\frac{2 \pi}{3}} \Psi=\exp ( \pm i 2 \pi / 3) \Psi\right]$, this is the first family of levels (labeled $3-$ in the following) that could sustain chiral states $\left(\mathcal{R}_{2 \pi / 3}\right.$ is a spatial rotation of angle $\left.2 \pi / 3\right)$. The last family of excited states (open triangles) belongs to the non trivial IR of $C_{3 v}$, the states invariant under a rotation of $2 \pi / 3$ and odd under axial symmetry $\left(\sigma_{x}\right)\left[\mathcal{R}_{\frac{2 \pi}{3}} \Psi=\Psi, \sigma_{x} \Psi=-\Psi\right]$ (labeled $3=x-$, see table III).

For larger $N$, the spectra become more and more dense, but this hierarchy of levels is always obeyed. The magnon states collapse to the ground-state roughly as $k$ i.e. as $N^{-1 / 2}$ 
whereas the energy of the chiral states seems to have a gap $\left(E_{3-}-E_{0}=6,6,1.908,2.958\right.$, $0.7661,2.454,2.641,2.01,2.546$, for $N=7,9,12,13,16,19,21,25,27)$. The hypothesis of a chiral ground-state on the triangular lattice seems excluded by our results [40,41]. The energies of the $3=x$ - states are much higher in the spectra (out of the range of energies of table I).

\section{QUALITATIVE ANALYSIS OF THE QUASI-DEGENERATE GROUND-STATE MULTIPLICITY}

The symmetries and the dynamics of the QDJS are essential to understand the nature of the order in the thermodynamic limit. Let us begin our investigation by the symmetry analysis of the eigenspectrum.

\section{A. The QDJS as the coupling of three spins}

It is straightforward to verify (see for example Table $\mathbb{\text { q) }}$ ) that the QDJS family exhibits the exact number of states expected from the coupling of three spins of length $N / 6$ :

$$
N_{S}=\min (2 S+1, N / 2-S+1)
$$

This number is readily obtained by noticing that the Hilbert space of two spins $S_{A}=S_{B}=$ $N / 6$ can be split in $(N / 3+1)$ subspaces associated to the eigenvalues of their sum $S_{A+B}$

(with $\left|S_{A}-S_{B}\right|=0 \leq S_{A+B} \leq S_{A}+S_{B}=N / 3$ ). The coupling of $S_{A+B}$ with $S_{C}$ gives then a total spin $\mathrm{S}\left(\left|S_{A+B}-N / 6\right| \leq S \leq S_{A+B}+N / 6\right)$, which leads to result Eq.2.

Such a property is not unexpected if we look at that family of levels as arising from the renormalization of the classical Néel state by quantum fluctuations. In this purpose it is most useful to Fourier analyze the hamiltonian Eq.1; separating the $\mathbf{k}=\mathbf{0}$ and $\pm \mathbf{k}_{0}$ contributions from the others, Eq.11 reads:

$$
\mathcal{H}=\mathcal{H}_{0}+\mathcal{V}
$$

where

$$
\mathcal{H}_{0}=\frac{9}{N}\left(\mathbf{S}^{2}-\mathbf{S}_{A}^{2}-\mathbf{S}_{B}^{2}-\mathbf{S}_{C}^{2}\right)
$$


$\mathbf{S}_{A}\left(\right.$ resp. $\left.\mathbf{S}_{B}, \mathbf{S}_{C}\right)$ is the total spin of the $A($ resp. $B, C$ ) sublattice, and

$$
\mathcal{V}=\sum_{k \neq 0 ; \pm k_{0}} f(\mathbf{k}) \mathbf{S}_{\mathbf{k}} \cdot \mathbf{S}_{-\mathbf{k}}
$$

with

$$
\mathbf{S}_{\mathbf{k}}=\frac{1}{\sqrt{N}} \sum_{i} \mathbf{s}_{i} \exp \left(i \mathbf{k} \cdot \mathbf{r}_{i}\right)
$$

and

$$
f(\mathbf{k})=\exp \left(i \mathbf{k} \cdot \mathbf{u}_{1}\right)+\exp \left(i \mathbf{k} \cdot \mathbf{u}_{2}\right)+\exp \left(-i \mathbf{k} \cdot\left(\mathbf{u}_{1}+\mathbf{u}_{2}\right)\right)+c . c
$$

where $\mathbf{u}_{1}$ and $\mathbf{u}_{2}$ are the two basis vectors of the lattice.

$\mathcal{H}_{0}$ commutes with $\mathbf{S}_{A}, \mathbf{S}_{A}^{2}, \mathbf{S}_{B}, \mathbf{S}_{B}^{2}, \mathbf{S}_{C}, \mathbf{S}_{C}^{2}$ and the eigenstates of $\mathcal{H}_{0}$ (written as $\left.\mid \Psi_{0}\left(i, S, M_{S}\right)>\right)$ are eigenstates of $\mathbf{S}^{2}, \mathbf{S}_{A}^{2}, \mathbf{S}_{B}^{2}, \mathbf{S}_{C}^{2}$ with eigenvalues:

$$
E\left(S, S_{A}, S_{B}, S_{C}\right)=\frac{9}{N}\left(S(S+1)-S_{A}\left(S_{A}+1\right)-S_{B}\left(S_{B}+1\right)-S_{C}\left(S_{C}+1\right)\right) .
$$

For each $S$ value the lowest eigenstates of $\mathcal{H}_{0}$ are obtained for $S_{A}=S_{B}=S_{C}=\frac{N}{6}$, their energies are:

$$
E_{0}(S)=-\frac{3}{4}(N+6)+\frac{9}{N} S(S+1) .
$$

These states $\mid \Psi_{0}^{0}\left(i, S, M_{S}\right)>$ (with $i$ from 1 to $N_{s}$ ), fully polarized on each magnetic sublattice, are the projections of the classical Néel states on the various IRs of $S U(2)$. They present the usual $2 S+1$ degeneracy associated to $M_{S}$ multiplied by the number $N_{S}$ of different couplings of three spins $N / 6$ (Eq.2).

As $\mathbf{S}_{A}^{2}, \mathbf{S}_{B}^{2}, \mathbf{S}_{C}^{2}$ do not commute with $\mathcal{V}, \mid \Psi_{0}^{0}\left(i, S, M_{S}\right)>$ are not eigenstates of $\mathcal{H}$, but we can look at them as the first (bad) approximation to the exact QDJS: the perturbation $\mathcal{V}$ dresses these "classical states" with quantum fluctuations, decreasing the average value of the sublattice magnetizations and lowering their energy towards the exact results. This process can lift the $N_{S}$ degeneracy of the $\left|\Psi_{0}^{0}\left(i, S, M_{S}\right)\right\rangle$ and it is indeed what is observed in the exact spectra of QDJS, but we verify in fig.2 and Table 1 that the set of QDJS has exactly the correct multiplicity $N_{S}$. 


\section{B. Symmetries of the QDJS}

The QDJ eigenstates belong to the three following IRs of the space symmetry group:

$\Gamma_{1}:\left[\mathbf{k}=\mathbf{0}, \mathcal{R}_{\pi} \Psi=\Psi, \mathcal{R}_{2 \pi / 3} \Psi=\Psi, \sigma_{x} \Psi=\Psi\right], \Gamma_{2}:\left[\mathbf{k}=\mathbf{0}, \mathcal{R}_{\pi} \Psi=-\Psi\right.$, $\left.\mathcal{R}_{2 \pi / 3} \Psi=\Psi, \sigma_{x} \Psi=\Psi\right]$ and $\Gamma_{3}:\left[\mathbf{k}= \pm \mathbf{k}_{0}, \mathcal{R}_{2 \pi / 3} \Psi=\Psi, \sigma_{x} \Psi=\Psi\right]$, where $\mathcal{R}_{\phi}$ is a rotation of angle $\phi$ and $\sigma_{x}$ is an axial symmetry.

They appear with regular rules (described below in III.D and in Appendix B) in all the IRs of $\mathrm{SU}(2)$ : that is for each $S$ value.

This proves that the QDJ eigenstates are invariant:

1) under translations of the magnetic sublattices. They only contain the $\mathbf{k}=\mathbf{0}$ or $\pm \mathbf{k}_{0}$ IRs of the lattice translation operators.

2) under the point group $C_{3 v}\left(\mathcal{R}_{2 \pi / 3} \Psi=\Psi, \sigma_{x} \Psi=\Psi\right)$.

The appearance of the $\mathbf{k}=\mathbf{0}$ and $\pm \mathbf{k}_{0}$ IRs of the translation group in this quasidegenerate ground-state multiplicity allows to build states which breaks the translational symmetry of the lattice (as it is the case of the Néel state). In the same approach, the appearance of all the IRs of $C_{2}$ (states where $\mathcal{R}_{\pi} \Psi= \pm \Psi$ ) allows to break the inversion symmetry, whereas the presence of all IRs of $S U(2)$ allows to build states with a vectorial magnetization pointing in a given direction. In summary, all the IRs which keep invariant the Néel states appear in the QDJS and no others. Note that the low lying levels of $\mathcal{H}_{0}$ described in the previous subsection belong to the same IRs than the QDJS. It is straightforward to verify that the quantum perturbation $\mathcal{V}$ is also invariant under the symmetry group of the classical Néel states; thus the renormalization by $\mathcal{V}$ of the sublattices spins can take place without disturbance of the symmetries of the classical Néel states.

The picture of the Néel states as linear combinations of the QDJS becomes plausible. But the symmetry argument does not prove by itself that the low lying levels of $\mathcal{H}_{0}$ have evolved identically in the dressing by the quantum fluctuations induced by $\mathcal{V}$. For example, the simple question "do all the QDJS have the same extensive sublattice magnetization?" cannot be answered from only symmetry arguments. A still more basic issue is discussed in the following subsection. 


\section{The effective dynamics of the QDJS}

We have shown in the previous subsections that the QDJS possess the same space symmetries than the (sphericalized) classical Néel states. We have now to make sure that the dynamics of this set of states can be reduced in the thermodynamic limit to that of a collective variable: the order parameter of the antiferromagnet. This is indeed a very serious issue and the necessary condition for the rigidity of the supposed-to-be ordered phase.

To precise the nature of this order parameter, let us concentrate on a presumed Néel order on the triangular lattice. Defining a specific Néel state requires the knowledge of exactly three angles: two angles locate the helicity $\Upsilon$ defined as:

$$
\Upsilon=\sum_{<i, j, k>}\left(\mathbf{s}_{\mathbf{i}} \wedge \mathbf{s}_{\mathbf{j}}+\mathbf{s}_{\mathbf{j}} \wedge \mathbf{s}_{\mathbf{k}}+\mathbf{s}_{\mathbf{k}} \wedge \mathbf{s}_{\mathbf{i}}\right)
$$

where the sum is taken on upward triangles in the counter-clockwise direction, the third angle locates the direction of the magnetization of one sublattice. In this paper we shall call (3) the direction of the helicity, (1) the direction of the $A$-sublattice magnetization and (2) the third orthogonal direction. When the reference to a laboratory frame will be necessary, we shall add a prime to the Néel-axes frame indices, keeping the unprimed quantities for the laboratory frame. In the Néel ground-states (and in their first long-wavelength excitations) the length of the sublattices magnetization is supposed to be constant; thus, the orientations of the Néel frame are the only variables of the problem (homogeneous on the lattice in the ground-states and slowly spatially variable in the first excitations): in other words the order parameter is an element of $S O(3)$. Let us now consider the dynamics of this collective variable in the homogeneous Néel state. On a finite lattice, this collective variable has a finite "inertia" and its free dynamics is entirely determined by its angular nature and the isotropy of spin space. We thus expect it to be that of a free top. Such a dynamics is at best described in the frame of principal axes of the object. The planar symmetry of the Néel state implies that one of the principal axes will be directed perpendicular to the plane of the spins, that is parallel to the helicity $\Upsilon$. In the principal axes of the Néel state, the hamiltonian describing the free dynamics of the system reads:

$$
H_{\text {eff }}=\frac{S_{1}^{2}}{2 I_{1}}+\frac{S_{2}^{2}}{2 I_{2}}+\frac{S_{3}^{2}}{2 I_{3}}
$$


where $S_{1}, S_{2}, S_{3}$ are the three components of the total spin of the system and $I_{1}, I_{2}, I_{3}$ the principal moments of inertia. $I_{1}, I_{2}, I_{3}$ are indeed linear responses to homogeneous magnetic fields in the spin plane $\left(I_{1}, I_{2}\right)$ or perpendicular to it $\left(I_{3}\right)$. They are the extensive homogeneous susceptibilities of this system. In the thermodynamic limit, we expect $I_{1}$ and $I_{2}$ to be equal by symmetry, as they are associated to magnetic fields which rotate the spins out of the plane: we denote them $I_{\perp} \equiv N \chi_{\perp}$, and $I_{3}$ is denoted $I_{\|} \equiv N \chi_{\|}$, where $\chi_{\perp}$ and $\chi_{\|}$are the perpendicular and parallel susceptibilities. For the classical Heisenberg model, $\chi_{\perp}=\chi_{\|}=1 / 18$ (see Eq.99). Nevertheless, it is likely that on the triangular lattice the renormalization of the two quantities by quantum fluctuations can be different 42 45. Thus, we shall focus on the dynamics of a symmetric top.

All the considerations up to now lay on the angular nature of the collective variable and are valid in a classical as well as in a quantum mechanics point of view. The quantization rules can be obtained from elementary quantum mechanics, but it is interesting to look first at the classical approach of the dynamics of this top [46]. The free dynamics of a top is entirely described by the relative motion of three directions (see fig.5): the total angular momentum $\mathbf{S}$ (a conserved quantity which is fixed in the lab frame), the angular rotation vector $\Omega$ and the principal axis of inertia of the top (axis $\mathbf{3}^{\prime}$, directed along the helicity of the Néel state). For a free symmetric top, these three directions are in fact always coplanar, and the global motion is the combination of two rotations: the precession of axis $\mathbf{3}^{\prime}$ of the top around the total angular momentum $\mathbf{S}$ with an angular velocity $\Omega_{p r}=S / I_{1}$ and the uniform spinning of the top on itself around $\mathbf{3}^{\prime}$ with the angular velocity $\Omega_{\mathbf{3}^{\prime}}=S_{\mathbf{3}^{\prime}} / I_{3}=S \cos \theta / I_{3}$. The dynamics reduces to two separate motions of two angular variables constrained to vary on $[0,4 \pi](S U(2)$ variables $)$. In quantum mechanics, these two angular constraints imply the quantizations of the eigenstates of the associated rotation generators. The first condition provides us trivially with the quantization of the total angular momentum, and its $(2 S+1)$ degeneracy. The second condition is not so trivial and implies the quantization of the projection of the total spin on the $\mathbf{3}^{\prime}$ axis of the top (not to be confused with the projection of the total spin on the 3 lab-frame axis). For a given $S$ value, $S_{3^{\prime}}$ can thus take $2 S+1$ values ranging from $S$ to $-S$. The hamiltonian then appears in the canonical form:

$$
H_{\mathrm{eff}}=\frac{\mathbf{S}^{2}}{2 I_{\perp}}+S_{3^{\prime}}^{2}\left(\frac{1}{2 I_{\|}}-\frac{1}{2 I_{\perp}}\right)
$$


The degeneracy of the eigenlevels of Eq.12 is $(2 S+1)$ for $S_{3^{\prime}}=0$ and $2 \times(2 S+1)$ for $S_{3^{\prime}} \neq 0$; for a given $S$ value of the total momentum the dimension of the Hilbert space is $(2 S+1)^{2}$.

Thus the rigidity of the ordered Néel states, implies that the low lying spectrum of Eq.1 must map in the thermodynamic limit, on the spectrum of Eq.12: that is indeed a very striking feature of finite-size spectra displayed in fig. 4 and 6 where both the leading behavior of Eq.12 and the global multiplicity $(2 S+1)^{2}$ of the top is well verified up to a total spin $S=N / 6$ (for higher values of $S$, the multiplicity $(2 S+1) N_{S}\left(N_{S}\right.$ given by Eq.2) is lower than $\left.(2 S+1)^{2}\right)$. The inertia $I_{\perp}$ in Eq.12 is indeed an extensive quantity scaling as $N$ (see fig.3). This leads to a determination of $\chi_{\perp}=I_{\perp} / N$ which is not very different, and only a bit smaller than the first-order spin-wave calculations of Chubukov et al. [45] (see fig.7a).

The situation is more difficult as regard the precise determination of the anisotropy and of $\chi_{\|}$. For the small samples studied in this work, the size effects are still extremely important: for $N=9$ the top is spherical, for $N=12$ no definite sign can be ascribed to $\left(\frac{1}{2 I_{\|}}-\frac{1}{2 I_{\perp}}\right)$. For the two larger samples $(N=21,27)$ there is a tendency towards a behavior of an oblate top $\left(\frac{1}{2 I_{\|}}-\frac{1}{2 I_{\perp}}\right)<0$, but the expected degeneracies of the symmetric top are not present leading to large uncertainties on $\chi_{\|}$(see fig.6) . Unfortunately the Hilbert space of the quantum top in the $N=36$ case is too large to allow the determination of the complete spectrum of low lying levels and we are thus unable to decide clearly even on the sign of the above quantity in this case.

\section{Spatial symmetries of the quantum effective top}

We have determined in subsection III.B the three IRs characterizing Néel order on the triangular lattice:

$\Gamma_{1}:\left[\mathbf{k}=\mathbf{0}, \mathcal{R}_{\pi} \Psi=\Psi, \mathcal{R}_{2 \pi / 3} \Psi=\Psi, \sigma_{x} \Psi=\Psi\right], \Gamma_{2}:\left[\mathbf{k}=\mathbf{0}, \mathcal{R}_{\pi} \Psi=-\Psi, \mathcal{R}_{2 \pi / 3} \Psi=\Psi\right.$, $\left.\sigma_{x} \Psi=\Psi\right]$ and $\Gamma_{3}:\left[\mathbf{k}= \pm \mathbf{k}_{0}, \mathcal{R}_{2 \pi / 3} \Psi=\Psi, \sigma_{x} \Psi=\Psi\right]$.

These IRs should be identified with the three IRs of the invariance group $C_{3 v}$ of the magnetic arrangement. Each symmetry of the lattice results in a permutation of the magnetic sublattices. Because the order parameter is an element of $S O(3)$, such a transformation may be cleared by a global rotational symmetry of the spins. This property allows us to compute the number of occurrences of the three IRs (see Appendix B): 


$$
n_{\Gamma_{1}}=(a+3 b+2 c) / 6, \quad n_{\Gamma_{2}}=(a-3 b+2 c) / 6, \text { and } n_{\Gamma_{3}}=(a-c) / 3,
$$

where

$$
a=2 S+1, b=\cos (S \pi) \text { and } c=\sin \left(\frac{2 \pi}{3}(2 S+1)\right) / \sin \left(\frac{2 \pi}{3}\right) .
$$

In the hypothesis of an à la Néel symmetry breaking, the low energy spectrum of Eq.11 should thus contain for each $S, M_{S}$ subspace:

$$
\begin{aligned}
& n_{\Gamma_{1}}\left[\mathbf{k}=\mathbf{0}, \mathcal{R}_{\pi} \Psi=\Psi, \mathcal{R}_{2 \pi / 3} \Psi=\Psi, \sigma_{x} \Psi=\Psi\right] \mathrm{IR}, \\
& n_{\Gamma_{2}}\left[\mathbf{k}=\mathbf{0}, \mathcal{R}_{\pi} \Psi=-\Psi, \mathcal{R}_{2 \pi / 3} \Psi=\Psi, \sigma_{x} \Psi=\Psi\right] \mathrm{IR} \text { and } \\
& n_{\Gamma_{3}} \text { couples of degenerate }\left[\mathbf{k}= \pm \mathbf{k}_{0}, \mathcal{R}_{2 \pi / 3} \Psi=\Psi, \sigma_{x} \Psi=\Psi\right] \text { IRs. }
\end{aligned}
$$

This appears to be true in all the exact spectra that we have computed, for all $S$ values up to $N / 6$ (see Table @ and fig.6).

The mapping of the low lying levels of Eq.11 on those of Eq.12 would imply a "quasidegeneracy" of some $\Gamma_{1}$ and $\Gamma_{2}$ levels. This phenomenon is not present in the studied samples (see fig.60); this does not exclude the possibility of a quasi-degeneracy in the thermodynamic limit, but this explains the difficulty to extract $\chi_{\|}$from these data.

At that point, we have determined both the dynamics and the symmetries of the QDJS that should appear in exact spectra of a system exhibiting a Néel ordered phase in the thermodynamic limit. As regards these criteria the spectra of the THA point in favor of a Néel ground-state: the scaling of $I_{\perp}$ seems even to dismiss the case of quantum criticality ( $I_{\perp}$ should then scale as $N^{1 / 2}$ as shown by Azaria et al 434). We shall come back to this point in the conclusion.

\section{E. Spectra of samples with $N=3 p+1$}

The periodic samples which do not frustrate Néel order, must have three sublattices invariant in the periodic boundary conditions, that is a number of spins $N=3 p$. On the nowadays computers and algorithms, memory requirements limit the studies to samples of $N=9,12,21,27$ and 36 . In order to enlarge the number of data available and have a complementary check of our hypothesis we have thus relaxed the periodic boundary conditions to allow the studies of the $7,13,16,19,25$ and 28 samples. Let us look at such a tiling of the infinite lattice (see fig.8). In order to preserve the possibility of a three lattices symmetry 
breaking on the infinite lattice, the translations operations $\mathbf{T}_{1}$ and $\mathbf{T}_{2}$ should be linked to a rotation of the spins: to be specific, in fig.8, the interaction between the spin (10) and (1') is chosen to be equal to the interaction of (10) with the periodic image of $(1)$ rotated by $2 \pi / 3$ and so on. Such boundary conditions, which imply the selection of an axis of rotation, break the rotational symmetry of the hamiltonian and the total spin is no longer a good quantum number, but the component $S_{3}$ of the total spin on the axis of rotation remains a conserved quantity. If we keep a global reference frame for the spins, the translational symmetry is difficult to handle. But this essential symmetry becomes again trivial by the choice of local frames rotated by $\pm 2 \pi / 3$ (resp. $\mp 2 \pi / 3$ ) in one-step translations of $\mathbf{u}_{1}$ (resp. $\mathbf{u}_{2}$ ). Within such a choice the Heisenberg hamiltonian reads

$$
\mathcal{H}=2 \sum_{<i, j>} \tilde{\mathbf{s}}_{i} \cdot \mathcal{R}_{3}^{-1}(2 \pi / 3) \tilde{\mathbf{s}}_{j} \mathcal{R}_{3}(2 \pi / 3)
$$

where $\tilde{\mathbf{s}_{i}}, \tilde{\mathbf{s}_{j}}$ are the spin operators in the local reference frames and $\mathcal{R}_{3}(2 \pi / 3)=$ $\exp \left(-i \pi / 3 \sigma_{3}\right), \sigma_{3}$ being the third Pauli matrix. We can in fact solve this problem for any value $(\phi, \psi)$ of the rotations associated to one step translations in the $\mathbf{u}_{1}$ and $\mathbf{u}_{2}$ directions (as long as the total rotation around a plaquette is zero). We have indeed done it and verified that the absolute minimum of the energy is obtained for the values $( \pm 2 \pi / 3)$ for samples with $N=3 p+1$ and for 0 and $\pm 2 \pi / 3$ for samples with $N=3 p$ (see fig.9). The same method gives information on the spin stiffnesses of the system [47].

If the system has Néel order at $T=0$, the spectrum of the low lying levels of Eq.14 is easily deduced from the general considerations of section III.C. The present boundary conditions induce a cylindrical symmetry of the problem around the $\mathbf{3}$ axis of the laboratory: this implies that the helicity axis $\mathbf{3}^{\prime}$ of the Néel state (see fig.5) is now constrained to coincide with the $\mathbf{3}$ axis of the laboratory. There is only one degree of freedom left, associated with the rotations around this axis, and one constant of motion: the $S_{3}$ component of the total spin. The effective hamiltonian Eq.11 in these conditions reduces to:

$$
\mathcal{H}_{\text {eff }}=\frac{S_{3}^{2}}{2 I_{3}}=\frac{S_{3}^{2}}{2 N \chi_{\|}}
$$

the two other terms in Eq.11 not related to a constant of motion should average to zero in any eigenstates. In our problem, $S_{3}$ can take all the values ranging from $-N / 2$ up to $N / 2$ and the degeneracy of each eigenstates of Eq.15 is 2 for $S_{3} \neq 0$ and 1 for $S_{3}=0$. 
Diagonalization results entirely corroborate these deductions as can be seen in fig.10. The analysis of the low lying levels obeying Eq.15 gives a determination of $\chi_{\|}$(see fig.7]b). As for the $N=3 p$ case, the values obtained by this method are in agreement and only a bit larger than the first-order spin-wave approximation, moreover the size effects seem to be roughly the same in the two approaches.

\section{F. Extrapolation of the ground-state energy per bond}

For ordered systems, the finite-size effect on the $N$-ground-state is mainly due to the cut-off of the long wave-length excitations. In the supposed-to-be Néel order these excitations are magnons, linear in $k$ and the leading term of the finite-size corrections to the ground-state energy is of order $N^{-1 / 2}$ and thus the ground-state energy per bond varies as $N^{-3 / 2}$ : more sophisticated approaches would allow to compute the first coefficients of these expansions as function of the spin-wave velocities 36,48,43. In fact in the previously developed theories [42,43] only the $N=3 p$ samples have been taken into consideration: in this case the fluctuations associated to the three modes of magnons equally contribute to the renormalization. In the $N=3 p+1$ samples, the approach to the singular points at $\mathbf{k}=\mathbf{0}$ and $\pm \mathbf{k}_{0}$ is different and we expect different coefficients in the $N^{-\alpha}$ expansion. It is effectively what is seen in the finite-size scaling analysis of the spin-wave susceptibilities (fig.7) and of the spin-wave energies (fig.11).

The analysis of the diagonalization results is more subtle, because it depends on the parity of $N$. The ground-state is either an $S=0$ or an $S=1 / 2$ state and in this latter case one has to take into account the top inertial effects described in the previous subsections. This leads to an $S / N$ correction to the ground-state energy (an $S / N^{2}$ correction to the energy

per bond) which is noticeable for the small sizes considered here. In order to compare the exact results to finite-size spin-wave results, it is necessary to extrapolate the exact $E(S, N)$ to an effective value $E(0, N)$. Two estimates of $E(0, N)$ can be computed. The first one is obtained by the subtraction from the ground-state energy of the main inertial contribution of $<\Psi_{0}\left|\mathcal{H}_{\mathrm{eff}}\right| \Psi_{0}>$ that is for $N=3 p$ samples:

$$
<2 \mathbf{s}_{i} \cdot \mathbf{s}_{j}>_{0, N}^{[1]}=<\Psi_{0}\left|2 \mathbf{s}_{i} . \mathbf{s}_{j}\right| \Psi_{0}>-<\Psi_{0}\left|\frac{\mathbf{S}^{2}}{6 N^{2} \chi_{\perp}}\right| \Psi_{0}>
$$


and for $N=3 p+1$ samples

$$
<2 \mathbf{s}_{i} \cdot \mathbf{s}_{j}>_{0, N}^{[1]}=<\Psi_{0}\left|2 \mathbf{s}_{i} \cdot \mathbf{s}_{j}\right| \Psi_{0}>-<\Psi_{0}\left|\frac{S_{3}^{2}}{6 N^{2} \chi_{\|}}\right| \Psi_{0}>.
$$

The second estimate is an average of the same quantity taken on all the QDJS up to $S=N / 6$

$$
<2 \mathbf{s}_{i} \cdot \mathbf{s}_{j}>_{0, N}^{[2]}=1 / N_{Q D J S} \sum_{S=m i n}^{S=N / 6}<Q D J S\left|\frac{\mathcal{H}-\mathcal{H}_{\text {eff }}}{3 N}\right| Q D J S>
$$

where $\mathcal{H}$ is the Heisenberg hamiltonian Eq.1 and $\mathcal{H}_{\text {eff }}$ is given by Eq.12 or Eq.15 according to the number of spins in the samples.

The first estimate (used in [33]) is different from the exact ground-state for odd $\mathrm{N}$ samples only, whereas the second estimate which is an average over a large number of levels, always differ from the exact ground-state. These averaged results are compared to finite-size spinwave results (see fig.11) where it is seen that the exact results are lower than the spin-wave results, the finite-size effects on the two sets of data being nevertheless roughly the same. It has been shown by Azaria and coworkers that the finite-size correction to the ground-state energy of the $N=3 p$ samples is 43 :

$$
E_{N}=E_{\infty}-\alpha\left(c_{\|}+2 c_{\perp}\right) N^{-3 / 2}
$$

where $\alpha$ is a geometrical form factor and $c_{\|}, c_{\perp}$ are in plane and out of plane spin-wave velocities.

On the basis of our numerical data we cannot decide on the exact value of the renormalized spin-wave velocities: it does not seem to be largely different from the spin-wave results, both in the $N=3 p$ (fig.11]a) and in the $N=3 p+1$ cases (fig.11]). The complete set of data analyzed within the following hypothesis

$$
c_{\|}=c_{\| s w} \quad c_{\perp}=c_{\perp s w}
$$

gives an estimate of the energy per bond in the thermodynamic limit

$$
<2 \mathbf{s}_{i} \cdot \mathbf{s}_{j}>_{\infty}=-0.363
$$

(see fig.11c).

At the end of this section, we can conclude that the spectral properties of the Heisenberg hamiltonian give strong argument in favor of an ordered ground-state and that the numerical 
spectral data and finite-size corrections are consistent with the picture and not very different from the spin-wave results. We will now discuss the important issue of the value of the order parameter of these Néel states.

\section{ORDER PARAMETER OF THE NÉEL STATES}

The check for long range order on the lattice could involve the measurement of the twopoints correlations $<\mathbf{s}_{i}(0) . \mathbf{s}_{j}(r)>$ or that of a macroscopic observable as the sublattice magnetization.

In view of the lattice sizes that can be studied, the analysis of the two-points correlation functions is rather unconclusive, the asymptotic behavior is far from reached and the sizes are still too small to check the Kennedy-Lieb-Shastry inequality [49. The measurement of the squared sublattice magnetization $\mathcal{M}^{2}$ :

$$
\mathcal{M}^{2}=<\mathbf{S}_{A}^{2}>=<\mathbf{S}_{B}^{2}>=<\mathbf{S}_{C}^{2}>
$$

or of the static structure factor $\mathcal{S}(\mathbf{k})$ defined in state $\mid \Psi>$ as

$$
\mathcal{S}(\mathbf{k})=<\Psi\left|\mathbf{S}_{-k} \cdot \mathbf{S}_{k}\right| \Psi>
$$

(with $\mathbf{S}_{k}$ in Eq.6) is easier. $\mathcal{S}(\mathbf{k})$ measured in the ground-state of the $N=3 p$ samples is shown in fig.12. The signature of Néel order in the $N$-ground-state should appear as a macroscopic value of $\mathcal{M}$ or $\mathcal{S}\left(\mathbf{k}_{\mathbf{o}}\right)$ using the following identity:

$$
2 N \mathcal{S}\left(\mathbf{k}_{0}\right)+<\Psi\left|\mathbf{S}^{2}\right| \Psi>=9 \mathcal{M}^{2}
$$

It should be kept in mind that, in the studied samples, the largest sublattices spins range from $3 / 2$ (for $N=9$ ) to 6 (for $N=36$ ): they cannot be treated as classical quantities. $\mathcal{M}^{2}$ is the square of a vector, it should be normalized by its maximum quantum value $(N / 6)(N / 6+1)$ (reached in the fully aligned classical Néel state $\mid \Psi_{0}^{0}\left(i, S, M_{S}\right)>$ of subsection III.A). The structure factor (same tensorial form as $\mathcal{M}^{2}$ ) should be identically normalized. Two definitions of the order parameter $(X$ or $Y)$ seem equally valid:

$$
X^{2}=\frac{36 \mathcal{M}^{2}}{N(N+6)}, \quad \text { or } \quad Y^{2}=8 \frac{\mathcal{S}\left(\mathbf{k}_{0}\right)}{N+6},
$$


$X$ saturates at one in the classical Néel state (and in the $N=9$ sample where the quantum fluctuations are ineffective to reduce the magnetization sublattices) and should be decreased by the quantum fluctuations in the quantum ground-state円. For the $N=3 p+1$ samples, it is impossible to define a sublattice magnetization. On the other hand one can measure the sum $\overrightarrow{\mathcal{M}}_{t}$ of the magnetizations computed in the local basis: for a fully aligned Néel state the square of this vector should saturate to $(N / 2)(N / 2+1)$. We thus define in this case:

$$
X^{2}=\frac{4 \mathcal{M}_{t}^{2}}{N(N+2)}
$$

Finite-size scaling analysis indicates that the leading correction to these parameters should go as $N^{-1 / 2}$; analysis of the results (see fig.13) shows that for these small values of $N$ the subleading correction is important: the $N \rightarrow \infty$ extrapolation is thus rather difficult, but an extrapolation to a zero value seems highly improbable.

On the other hand, the comparison between the diagonalization results and first-order spin-wave results is interesting: it is seen in fig. 13 that the two sets of results do not differ by large amounts. It should be noted that Miyake spin-wave results are in our point view incorrect for finite sizes [17]. We agree with Deutscher and Everts formula for first-order spin-wave results which restrict the renormalization by quantum fluctuations to $\mathbf{k} \neq 0, \pm \mathbf{k}_{0}$ wave vectors [50]. As it is well known the spin-wave hamiltonian cannot be bosonized for $\mathbf{k}=0, \pm \mathbf{k}_{0}$ (the Bogolioubov transformation becomes singular). It is a bit lengthy but straightforward to show that these three Fourier components of the spin-wave hamiltonian

\footnotetext{
${ }^{1}$ Examination of this problem of normalization has been underestimated in previous studies [27.32]. Because of an erroneous normalization most of the weight of the extrapolation procedure has been carried on unphysical numbers larger than the saturation value of the fully aligned classical Néel state. This essentially explains the difference in the conclusions of the previous authors and ourselves as regard the question of order of the THA. Deutscher and Everts have been faced with the same kind of difficulty in their recent work on the $J_{1}-J_{2}$ hamiltonian on the triangular lattice [50]; by studying the behavior of the system when $J_{2} / J_{1} \rightarrow-\infty$ they arrive at the same kind of renormalization to order $1 / N$ than ours. The above line of reasoning is probably more general.
} 
can be recombined to give the total spin $S^{2}$ and that they do not participate to the renormalization of the order parameter (such a remark has recently been developed by Zhong and Sorella in the case of the square lattice Heisenberg hamiltonian |51]). Careful examination of these singular terms shows that the first-order correction to the ground-state energy is exactly obtained by the summation of the usual formula on the $N$ points of the Brillouin zone:

$$
<2 \mathbf{s}_{i} \cdot \mathbf{s}_{j}>_{s w}=-1 / 4+\frac{1}{2 N} \sum_{\mathbf{k}}\left(\omega_{\mathbf{k}}-1\right)
$$

where $\omega_{\mathbf{k}}=\left(1-\gamma_{\mathbf{k}}\right)^{1 / 2}\left(1+2 \gamma_{\mathbf{k}}\right)^{1 / 2}$ and $\gamma_{\mathbf{k}}=f(\mathbf{k}) / 6$ (see Eq.7). On the other hand, the correction to the $\mathcal{M}_{z}$ magnetization involves exclusively the $\mathbf{k} \neq 0, \pm \mathbf{k}_{0}$ points of the Brillouin zone that is $N-3$ points in the $N=3 p$ samples and $N-1$ points in the $N=3 p+1$ samples.

$$
<\mathcal{M}_{z}>=\frac{1}{2}\left[1-\frac{1}{N} \sum_{\mathbf{k} \neq \mathbf{0}, \pm \mathbf{k}_{\mathbf{0}}}\left(\frac{1+\frac{\gamma_{\mathbf{k}}}{2}}{\omega_{\mathbf{k}}}-1\right)\right]
$$

The careful comparison between the exact results and the first-order spin-wave results leads us to conclude that the spin-wave approximation seems an extremely good quantitative approximation for the considered sizes; on the basis of the present data, it seems highly hazardous to adopt other estimate of the thermodynamic limit that the spin-wave results [16] I. In a previous paper [33, we have shown that, for a given size, the order parameter modulus is roughly the same in all the QDJS.

\section{CONCLUSION}

The thorough analysis of exact results of diagonalization of the Heisenberg hamiltonian on periodic samples with triangular symmetry has brought various pieces of information

\footnotetext{
${ }^{2}$ Definitions and comparison of order parameter in symmetry breaking solutions (spin-wave approximation) and symmetry non breaking one (exact diagonalizations) has given birth to many studies [35,52 55]. In this work, we take care to compare two parameters that are normalized to 1 in the absence of fluctuations and should be equal in the thermodynamic limit. The problem of the symmetry breaking will be studied in a forthcoming paper.
} 
both qualitative and quantitative.

The qualitative information emerge from the symmetry analysis of the spectra: this analysis shows a strict hierarchy of levels. The first family, degenerated to the absolute ground-state in the thermodynamic limit, contains all the quantum states and only those needed to build symmetry breaking Néel states with three sublattices magnetizations in a $C_{3 v}$ invariant configuration. Their dynamics can roughly be mapped on the dynamics of a quantum symmetric top as expected from general considerations on the nature of the order parameter in the Néel ordered states [? Understanding this family of levels from the coupling of three macroscopic spins explains why the multiplicity of this quasi-degenerate set of levels (the QDJS) is of order $N^{3}$ (in the square lattice case the multiplicity should be of order $N^{2}$ ) which gives in the thermodynamic limit an entropy of order $\log N$. Rather unexpectedly the present analysis does not give quantitative answers extremely different from first-order spin-wave results. On the basis of the coherence of the data concerning the symmetries of the QDJS, their dynamics, the energies, the spin susceptibilities, and order parameter of both the $N=3 p$ and $N=3 p+1$ samples and their size effects, one can only sustain the hypothesis of an "ordered ground-state" for these small samples. Indeed we have no information of the effect of softer quantum fluctuations with wavelengths larger than about 6 lattice sizes. The clusters expansions of Singh and Huse and the high temperature expansions of Elstner, Singh and Young point to a weaker order (if any?) than obtained in the first-order spin-wave calculations. In these two last methods the invoked clusters are smaller than our largest sizes but there are no bias due to periodic boundary conditions and the sampling of the quantum fluctuations is different from what is done in this work.

The present state of the art does not exclude that for larger sizes than those explored today quantum fluctuations drive the system towards criticality but there is in the small samples studied here no evidence of such a behavior.

\footnotetext{
${ }^{3}$ We should mention that the first exhibition of such a structure of low lying levels invoked by Anderson in his seminal paper of 1952 appears in a paper by Gross, Sanchez-Velasco and Siggia [34] that regrettedly escaped our vigilance in our preliminary report of 1992, indeed most of the theoretical hints were present in the first paper of this series.
} 


\section{Acknowledgements:}

We acknowledge many fruitful discussions with P. Azaria, B. Delamotte, D. Mouhanna, S. Sachdev, P. Sindzingre and A.P. Young. We have benefited from a grant of computer time at Centre de Calcul Vectoriel pour la Recherche (CCVR), Palaiseau, France.

\section{APPENDIX A: GROUP THEORY ON THE THA, NUMERICAL METHOD}

Here, we explain how to obtain the complete spectrum of the spin- $1 / 2$ THA, for the largest possible samples compatible with the triangular symmetry of the infinite lattice. The number of eigenstates increases as $2^{N}$ with $N$. Even for very small values of $N$, there is no stable numerical method to calculate directly all the eigenvalues of $\mathcal{H}$. The problems to be handled are memory space requirement, computer time, and degeneracy of states. These problems can be reduced by an intensive use of space and spin symmetries. Indeed, when all symmetries are accounted for, one can work in subspaces where eigenvalues are no more degenerate and better control on round off errors is obtained. These subspaces come from the decomposition of the Hilbert space according to the irreducible representations (IRs) of the symmetry group of the problem.

This symmetry group is the direct product of the space symmetry group of the lattice times the spin rotation group $S U(2)$. Thus, its IRs are the tensor products of space-group IRs times $S U(2)$ IRs. We first consider in details the space symmetry, then quickly the spin symmetries and explain how they are implemented in the numerical approach.

\section{The space symmetry group}

The spins stand on a triangular lattice defined by the basis vectors $\mathbf{u}_{1}$ and $\mathbf{u}_{2}$ (see

fig.8). On the infinite triangular lattice, the group $\mathcal{G}_{\infty}$ of symmetries, which leave the lattice sites globally invariant and keep the vicinity relations, is the semi-direct product of the translation group $\mathcal{T}_{\infty}$ times the point group $\mathcal{P}$. The translation group $\mathcal{T}_{\infty}$ has two generators: the translations of vector $\mathbf{u}_{1}$ and $\mathbf{u}_{2}$. The point group $\mathcal{P}$ consists of the symmetry transformations keeping a site invariant: its generators are the planar rotations $\mathcal{R}_{\pi}$ and $\mathcal{R}_{2 \pi / 3}$ and the axial reflection $\sigma_{x}$ with respect to $\mathbf{u}_{1}$ (see fig.8). The point group $\mathcal{P}$ is isomorphic to $\mathcal{C}_{6 v}\left(\right.$ or $\left.\mathcal{D}_{6}\right)$ and has twelve elements: six rotations and six reflections. 
In order to preserve the translation invariance and to reduce the number of degrees of freedom, periodic conditions are used, defined by the vectors $\mathbf{T}_{1}=l \mathbf{u}_{1}+m \mathbf{u}_{2}$ and $\mathbf{T}_{2}=$ $l^{\prime} \mathbf{u}_{1}+m^{\prime} \mathbf{u}_{2}$ (see fig.8). $\mathcal{R}_{\pi}$ is always a symmetry transformation of such a system. For $\mathcal{R}_{2 \pi / 3}$ to be a symmetry transformation of the periodic lattice, we chose $\mathbf{T}_{2}=\mathcal{R}_{\pi / 3} \mathbf{T}_{1}\left(l^{\prime}=-m\right.$; $\left.m^{\prime}=l+m\right)$. The number $N$ of sites per cell $\left(\mathbf{T}_{1}, \mathbf{T}_{2}\right)$ is thus given by:

$$
N=l^{2}+l m+m^{2}
$$

The system has an axial reflection when $l m=0$ or $l=m$. Therefore the point group is $\mathcal{P} \equiv \mathcal{C}_{6 v}$ (resp. $\mathcal{C}_{6}$ if there is no $\sigma_{x}$ axis) and the space group $\mathcal{G}_{N}=\mathcal{T}_{N} \wedge \mathcal{C}_{6 v}\left(\right.$ resp. $\left.\mathcal{T}_{N} \wedge \mathcal{C}_{6}\right)$ has $12 N$ (resp. $6 N$ ) elements. In the following, periodic samples with $N$ multiple of 3 are used.

Let $E_{N}$ be the space of wavefunctions for a system of $N$ spins. A wavefunction is a linear combination of configurations:

$$
\Psi=\sum_{c} \alpha_{c} \mid c>
$$

where a configuration $\mid c>$ is an element of the basis $\mathcal{B}_{N}=\{|\uparrow>,| \downarrow>\}^{N}$ of $E_{N}$. $E_{N}$ is a linear representation of $\mathcal{G}_{N}$. It can be expressed as a direct sum of IRs of $\mathcal{G}_{N}$. Summing the isomorphic IRs leads to a unique factorization in a direct sum of subspaces $E_{N, \rho}$ associated to the various IRs $\rho$ of $\mathcal{G}_{N}$. Each of these subspaces is invariant under $\mathcal{H}$. Because $\mathcal{G}_{N}$ is not commutative, some of the IRs have dimensions greater than one resulting in a degeneracy of $\mathcal{H}$ on $E_{N, \rho}$. Thanks to the rather simple structure of group $\mathcal{G}_{N}$, one can eliminate this degeneracy by working on some subspace $E_{N, \rho}^{\prime}$ associated to some one-dimensional IR $\rho$ of some subgroup $\mathcal{G}_{N, \rho}$ of $\mathcal{G}_{N}$.

By using $E_{N, \rho}^{\prime}$ instead of $E_{N, \rho}$, the eigenvalue multiplicities are divided by the dimension of $\rho$, which is also equal to the ratio of the cardinal of $\mathcal{G}_{N}$ to the cardinal of $\mathcal{G}_{N, \rho}$.

Let us first consider the case where there is no axial reflection: the point group $\mathcal{P}$ is an abelian cyclic group of six elements. Because $\mathcal{T}_{N}$ is abelian, its IRs are one-dimensional and are characterized by $\mathbf{k}$, a vector of the Brillouin zone. Thus, $E_{N}$ is factorized in $N$ subspaces $E_{N, \mathbf{k}}$. The group $\mathcal{P}$ acts on these vectors to form different stars. Two vectors $\mathbf{k}_{1}$ and $\mathbf{k}_{2}$ belong to the same star if it exists an element of $\mathcal{P}$ (here a rotation) which transforms $\mathbf{k}_{1}$ in 
$\mathbf{k}_{2}$. Because this transformation commutes with the hamiltonian, the eigenvalue spectra of $E_{N, \mathbf{k}_{1}}$ and $E_{N, \mathbf{k}_{2}}$ will be identical. For a fixed $\mathbf{k}$, let $\mathcal{P}_{\mathbf{k}}$ be the subgroup of $\mathcal{P}$ which keeps $\mathbf{k}$ invariant. This subgroup is abelian and all its IRs are one-dimensional. The cardinal of $\mathcal{P}_{\mathbf{k}}$ is 6 for $\mathbf{k}=0$. It is 3 for $\mathbf{k}= \pm \mathbf{k}_{0}\left( \pm \mathbf{k}_{0}\right.$ are the corners of the Brillouin zone); this occurs only when $N$ is multiple of 3 . It is 2 if $\mathbf{k}=-\mathbf{k} \neq 0$ (the middle of the side of the Brillouin zone) and this occurs only when $N$ is multiple of 4 . It is 1 for the other $\mathbf{k}$-vectors. The different IRs are listed in Table पII.

When there is an axial reflection $(l m=0$ or $l=m)$, the group $\mathcal{P}$ is generated by $\sigma_{x}$ and $\mathcal{R}_{\pi / 3}$. If the group $\mathcal{P}_{\mathbf{k}}$ is abelian, the previous construction is applied. If the group $\mathcal{P}_{\mathbf{k}}$ is not abelian, some of its IRs are two-dimensional. This is the case for $\mathbf{k}=0\left(\mathcal{P}_{\mathbf{k}}=\mathcal{P}=\mathcal{D}_{6}\right)$ and $\mathbf{k}=\mathbf{k}_{0}\left(\mathcal{P}_{\mathbf{k}}=\mathcal{D}_{3}\right)$. The abelian normal subgroups are respectively $\mathcal{C}_{6}$ and $\mathcal{C}_{3}$. The different IRs are listed in Table 【II].

For small systems, other accidental space symmetries can occur which imply new degeneracies. If they commute with all the previous space symmetries, the degenerate states appear in the same IR. Otherwhise, the degenerate states stand in different IRs.

\section{The spin symmetry group}

The hamiltonian of Eq.1 is invariant under global spin rotations: it commutes with $\mathbf{S}^{2}$, where $\mathbf{S}$ is the total spin. For spin-1/2, the symmetry group is $S U(2)$. The IRs are labeled by $S$.

\section{Numerical method}

A general wavefunction is defined by the knowledge of the set of $2^{N}$ coefficients $\left\{\alpha_{c}\right\}$ Eq.30. The first easy reduction of this set is to work in an eigenspace of $S_{z}$ : the basis size thus becomes $C_{N}^{N / 2-S_{z}}$. The next step is to work in a given $E_{n, \rho}^{\prime}$, characterized by the phase factors associated to each element of $\mathcal{G}_{N, \rho}$. One groups together the configurations in conjugate classes: $\mid c>$ and $\mid c^{\prime}>$ belong to the same class if it exists an element $X$ of $\mathcal{G}_{N, \rho}$ such that $\left|c^{\prime}>=X\right| c>$. So, $\alpha_{c^{\prime}}$ differs from $\alpha_{c}$ by a phase factor listed in Tables [1] or 피. The number of independent coefficients is therefore reduced by roughly a factor $N$ (translations) and at maximum by a factor $12 N$ (cardinal of $\mathcal{G}_{N}$ ). The memory requirement 
and computer time are reduced by the same amount.

Implementation of the $S U(2)$ symmetry is done via a projector technique by using the operator:

$$
\prod_{\substack{i=N / 2, N / 2-1, \ldots \\ i \neq S}} \frac{\mathbf{S}^{2}-i(i+1)}{S(S+1)-i(i+1)}
$$

where $\mathbf{S}^{2}$ is computed thanks to relation $\mathbf{S}^{2}=S_{z}^{2}+S_{z}+2 S_{-} S_{+}$and $S_{+}$and $S_{-}$are computed like $\mathcal{H}$. Applying $\mathbf{S}^{2}$ is therefore as fast as applying the hamiltonian, or so.

Lanczös method has been applied to diagonalize the hamiltonian in the subspace associated to each IR $\rho$. Working with classes instead of configurations insures that the vectors generated by Lanczös method stay in the same subspace. For small systems, the hamiltonian and the two operators $S_{+}$and $S_{-}$are tabulated; for large systems $(N=36)$, they have to be computed at each step, but vector components are stored in an order allowing application of these three operators to be vectorized. In any case, diagonal part of the hamiltonian is computed separately at once and stored. Additional details on the technical tricks will be given elsewhere.

The algorithm is as follows: $i$ ) first, for some IR, build the conjugate-class table and the phase-factor table; $i i)$ tabulate $\mathcal{H}, S_{+}$and $\left.S_{-} ; i i\right)$ choose a random initial vector and project it out in the desired subspace of $\left.S^{2} ; i v\right)$ apply the Lanczös algorithm; v) calculate the eigenvectors, correlations...

In fact, round-off errors propagate very quickly in this algorithm. In order to eliminate these errors, computed vectors are orthogonalized to all previous ones and projected out in the $S$-subspace. One can compute the dimension $m_{\rho}(S)$ of this subspace and verify that, after exactly $m_{\rho}(S)$ iterations, the modulus of the last vector is zero. This is a strong test of this algorithm.

\section{APPENDIX B}

Here, we compute the number of replica of each irreducible representation of the QDJ states if a Néel state occurs. The idea is to use some symmetries that keep invariant the classical Néel state. These symmetries are the compositions of the permutations of the sublattices times specific spin rotations. 
Thus, let us start with an a priori classical Néel state and its symmetry group. In our case, the Néel state is made of three sublattices as described in section II. Let us define the plan $(x y)$ of the classical Néel state and $z$ a perpendicular axis. The group which permutes the three sublattices is $S_{3}$ (isomorphic to the dihedral group $D_{3}$ ). After a permutation which exchanges the spins between the sublattices (giving an other Néel state), the initial Néel state is recovered if the spins are rotated simultaneously. For example, the permutation of two sublattices (say $A$ and $B$ ) times a spin rotation of axis parallel to the spins of the last sublattice (thus $C$ ) recovers the initial Néel state. Likewise, a cyclic permutation of the three sublattices times a spin rotation of $2 \pi / 3$ along the $z$-axis also recovers the initial Néel state. Thus, two (resp. three) sublattices permutations are associated to an half (resp. a third of a) turn around an axis in the (xy) plane (resp. around the $z$-axis).

In the quantum case, the permutation of the sublattice is one of the symmetry of the lattice (say, central symmetry for the two sublattice permutation and translation of one step for three sublattice permutation) times a spin rotation in $S U(2)$. The group of these spin rotations is the dihedral group $D_{3}$ for integer spins $(S O(3))$, and is a group of 12 elements

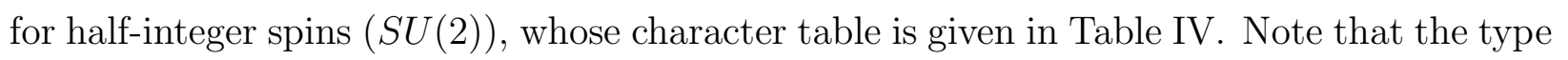
of spin rotation associated to each class is specific of the Néel state and is necessary (here also sufficient) to calculate the number of replicas $n_{\Gamma_{i}}$ of each irreducible representation (IR) $\Gamma_{i}$. As all the above transformations conserve the total spin $S$, one can thus calculate the traces of these rotations in the subspace of fixed $S, M_{S}$ :

$$
n_{\Gamma_{i}}^{(S)}=\frac{1}{6} \sum_{k} \operatorname{Tr}^{(S)}\left(\mathcal{R}_{k}\right) \chi_{i}(k) N_{e l}(k)
$$

For integer (resp. half-integer) spins, only the $n_{\Gamma_{i}}$ (resp. $n_{\Gamma_{i}^{\prime}}$ ) are non zero.

Now, we identify the $\Gamma_{i}$ IRs with the IRs of the hamiltonian. Because the QDJS have already been found to belong to three precise IRs (see section III.B), this identification is straightforward: $\Gamma_{0}$ is the trivial IR, $\Gamma_{1}$ is the IR odd with respect to the inversion $(\mathbf{k}=\mathbf{0}$, invariant under a $2 \pi / 3$ rotation), and $\Gamma_{3}$ is the last doubly degenerate representation $\left(\mathbf{k}=\mathbf{k}_{0}\right.$, invariant under a $2 \pi / 3$ rotation). The identification of the $\Gamma_{i}^{\prime}$ is the same for half-integer spins and leads to formulas (13) both for integer and half-integer spins. 


\section{REFERENCES}

[1] N.D. Mermin and H. Wagner, Phys. Rev. Lett. 17, 1133 (1966).

[2] P.W. Anderson, Phys. Rev. 86, 694 (1952).

[3] S. Liang, B. Doucot, and P.W. Anderson, Phys. Rev. Lett. 61, 365 (1988).

[4] D. Huse, Phys. Rev. B 37, 2380 (1988).

[5] J.D. Reger and A.P. Young, Phys. Rev. B 37, 5978 (1988).

[6] D.A. Huse and V. Elser, Phys. Rev. Lett. 60, 2531 (1988).

[7] S. Tang and J.E. Hirsch, Phys. Rev. B 39, 4548 (1989).

[8] M. Gross, E. Sanchez-Velasco, and E. Siggia, Phys. Rev. B 39, 2484 (1989).

[9] N. Trivedi and D. Ceperley, Phys. Rev. B 41, 4552 (1990).

[10] S. Liang, Phys. Rev. B 42, 6555 (1990).

[11] J. Carlson, Phys. Rev. B 40, 846 (1989).

[12] H. Schultz and T. Ziman, Europhys. Lett. 8, 355 (1992).

[13] P.W. Anderson, Mater. Res. Bull. 8, 153 (1973).

[14] P. Fazekas and P.W. Anderson, Philos. Mag. 30, 423 (1974).

[15] H. Nishimori and S.J. Miyake, Prog. Theor. Phys. 73, 18 (1985).

[16] T. Jolicoeur and J.C. Le Guillou, Phys. Rev. B 40, 2727 (1989).

[17] S.J. Miyake, J. Phys. Soc. Jpn. 61, 983 (1992).

[18] T. Oguchi, H. Kitatani, and H. Nishimori, J. Phys. Soc. Jpn. 56, 3858 (1987).

[19] R.R.P. Singh and D.A. Huse, Phys. Rev. Lett. 68, 1766 (1992).

[20] N. Eltsner, R.R.P. Singh, and A.P. Young, Phys. Rev. Lett. 71, 1629 (1993).

[21] P. Sindzingre, P. Lecheminant, and C. Lhuillier, preprint (unpublished). 
[22] T. Oguchi, H. Nishimori, and Y. Taguchi, J. Phys. Soc. Jpn. 55, 323 (1986).

[23] V. Kalmeyer and R.B. Laughlin, Phys. Rev. Lett. 59, 2095 (1987).

[24] K. Yang, L.K. Warman, and S.M. Girvin, Phys. Rev. Lett. 70, 2641 (1993).

[25] L.G. Marland and D.D. Betts, Phys. Rev. Lett. 43, 1618 (1979).

[26] S. Fujiki, Can. J. Phys. 65, 489 (1987).

[27] H. Nishimori and H. Nakanishi, J. Phys. Soc. Jpn. 57, 626 (1988).

[28] M. Imada, J. Phys. Soc. Jpn. 56, 311 (1987).

[29] M. Imada, J. Phys. Soc. Jpn. 58, 2650 (1989).

[30] T. Jolicoeur, E. Dagotto, E. Gagliano, and S. Bacci, Phys. Rev. B 42, 4800 (1990).

[31] R. Deutscher, H.V. Everts, S. Miyashita, and M. Wintel, J. Phys. A. Math. Gen. 23, L1043 (1990).

[32] P.W. Leung and K. Runge, Phys. Rev. B 47, 5861 (1993).

[33] B. Bernu, C. Lhuillier, and L. Pierre, Phys. Rev. Lett. 69, 2590 (1992).

[34] M. Gross, E. Sanchez-Velasco, and E. Siggia, Phys. Rev. B 40, 11328 (1989).

[35] T.A. Kaplan, P. Horsch, and W. von der Linden, J. Phys. Soc. Jpn. 58, 3894 (1989).

[36] H. Neuberger and T. Ziman, Phys. Rev. B 39, 2608 (1989).

[37] D.S. Fisher, Phys. Rev. B 39, 11783 (1989).

[38] E. Lieb and D. Mattis, J. of Math. Phys. 3, 749 (1962).

[39] B. Bernu, P. Lecheminant, C. Lhuillier, and L. Pierre, Physica Scripta 49, 192 (1993).

[40] G. Baskaran, Phys. Rev. Lett. 63, 2524 (1989).

[41] E.T. Tomboulis, Phys. Rev. Lett. 68, 3100 (1992).

[42] T. Dombre and N. Read, Phys. Rev. B 39, 6797 (1989).

[43] P. Azaria, B. Delamotte, and D. Mouhanna, Phys. Rev. Lett. 70, 2483 (1993). 
[44] A.V. Chubukov, T. Senthil, and S. Sachdev, Phys. Rev. Lett. 72, 2089 (1994).

[45] A.V. Chubukov, S. Sachdev, and T. Senthil, preprint (unpublished).

[46] L. Landau and E. Lifschitz, Mechanics (Pergamon Press, Oxford, 1960).

[47] P. Lecheminant, B. Bernu, C. Lhuillier, and L. Pierre, preprint (unpublished).

[48] P. Hasenfratz and F. Niedermayer, Z. Phys. B. Condensed Matter 92, 91 (1993).

[49] T. Kennedy, E.H. Lieb, and B.S. Shastry, J. Stat. Phys. 53, 1019 (1988).

[50] R. Deutscher and H.U. Everts, Z. Phys. B. Condensed Matter 93, 77 (1993).

[51] Q.F.Zhong and S. Sorella, Europhys. Lett. 21, 629 (1993).

[52] T. Koma and H. Tasaki, Phys. Rev. Lett. 70, 93 (1993).

[53] R.B. Griffiths, Phys. Rev. 152, 240 (1966).

[54] F.J. Dyson, E.H. Lieb, and B. Simon, J. Stat. Phys. 18, 335 (1978).

[55] H. Wagner, Z. Phys. B. Condensed Matter 195, 273 (1966). 
TABLES

\begin{tabular}{|c|c|c|c|c|c|c|c|c|}
\hline$N$ & $<2 \overrightarrow{\mathbf{s}}_{i} \cdot \overrightarrow{\mathbf{s}}_{j}>$ & $S$ & deg. & & k & $\mathcal{R}_{2 \pi / 3}$ & $\mathcal{R}_{\pi}$ & $\sigma$ \\
\hline \multirow[t]{13}{*}{9} & * -0.3888889 & 0.5 & 4 & 3 & -3 & 1 & 0 & 1 \\
\hline & -0.2777778 & 0.5 & 12 & 0 & 3 & 0 & 0 & -1 \\
\hline & -0.2777778 & 0.5 & 12 & 0 & 3 & 0 & 0 & 1 \\
\hline & * -0.2777778 & 1.5 & 4 & 0 & 0 & 1 & 1 & 1 \\
\hline & * -0.2777778 & 1.5 & 4 & 0 & 0 & 1 & -1 & 1 \\
\hline & $*-0.2777778$ & 1.5 & 8 & 3 & -3 & 1 & 0 & 1 \\
\hline & -0.1666667 & 0.5 & 4 & 0 & 0 & -1 & 1 & 0 \\
\hline & -0.1666667 & 0.5 & 8 & 3 & -3 & -1 & 0 & 0 \\
\hline & -0.1666667 & 0.5 & 12 & 0 & 3 & 0 & 0 & 1 \\
\hline & -0.1666667 & 1.5 & 24 & 0 & 3 & 0 & 0 & -1 \\
\hline & -0.1666667 & 1.5 & 24 & 0 & 3 & 0 & 0 & 1 \\
\hline & -0.0925926 & 2.5 & 6 & 0 & 0 & 1 & 1 & 1 \\
\hline & -0.0925926 & 2.5 & 12 & 3 & -3 & 1 & 0 & 1 \\
\hline \multirow[t]{15}{*}{12} & * -0.4068868 & 0 & 1 & 0 & 0 & 1 & 1 & 1 \\
\hline & * -0.3589325 & 1 & 6 & 4 & -4 & 1 & 0 & 1 \\
\hline & * -0.3570639 & 1 & 3 & 0 & 0 & 1 & -1 & 1 \\
\hline & -0.3538882 & 0 & 2 & 0 & 0 & -1 & 1 & 0 \\
\hline & -0.3382480 & 0 & 3 & 0 & 6 & 0 & -1 & 1 \\
\hline & -0.3370959 & 0 & 6 & 2 & 4 & 0 & 0 & 1 \\
\hline & * -0.3280998 & 1 & 9 & 0 & 6 & 0 & 1 & 1 \\
\hline & -0.3132131 & 1 & 18 & 2 & 4 & 0 & 0 & 1 \\
\hline & -0.3024839 & 1 & 9 & 0 & 6 & 0 & 1 & 1 \\
\hline & -0.2913787 & 0 & 1 & 0 & 0 & 1 & 1 & -1 \\
\hline & -0.2907218 & 0 & 4 & 4 & -4 & -1 & 0 & 0 \\
\hline & -0.2907008 & 1 & 18 & 2 & 4 & 0 & 0 & 1 \\
\hline & -0.2906078 & 0 & 1 & 0 & 0 & 1 & 1 & 1 \\
\hline & * -0.2862917 & 2 & 5 & 0 & 0 & 1 & 1 & 1 \\
\hline & -0.2827618 & 1 & 12 & 4 & -4 & -1 & 0 & 0 \\
\hline
\end{tabular}




\begin{tabular}{|c|c|c|c|c|c|c|c|c|}
\hline & * -0.2817881 & 2 & 10 & 4 & -4 & 1 & 0 & 1 \\
\hline & * -0.2694317 & 2 & 10 & 4 & -4 & 1 & 0 & 1 \\
\hline & -0.2693163 & 1 & 9 & 0 & 6 & 0 & 1 & -1 \\
\hline & -0.2585320 & 1 & 18 & 2 & 4 & 0 & 0 & 1 \\
\hline \multirow[t]{24}{*}{21} & * -0.3739972 & 0.5 & 4 & 7 & -7 & 1 & 0 & 0 \\
\hline & * -0.3516974 & 1.5 & 4 & 0 & 0 & 1 & 1 & 0 \\
\hline & * -0.3511989 & 1.5 & 8 & 7 & -7 & 1 & 0 & 0 \\
\hline & -0.3511315 & 0.5 & 12 & 3 & 9 & 0 & 0 & 0 \\
\hline & -0.3500572 & 0.5 & 12 & 1 & -4 & 0 & 0 & 0 \\
\hline & * -0.3499587 & 1.5 & 4 & 0 & 0 & 1 & -1 & 0 \\
\hline & -0.3435291 & 0.5 & 12 & 2 & -8 & 0 & 0 & 0 \\
\hline & -0.3402059 & 0.5 & 12 & 3 & 9 & 0 & 0 & 0 \\
\hline & -0.3392388 & 0.5 & 12 & 2 & -8 & 0 & 0 & 0 \\
\hline & -0.3390870 & 0.5 & 12 & 1 & -4 & 0 & 0 & 0 \\
\hline & -0.3320711 & 0.5 & 8 & 7 & -7 & -1 & 0 & 0 \\
\hline & -0.3296426 & 1.5 & 24 & 3 & 9 & 0 & 0 & 0 \\
\hline & -0.3292417 & 1.5 & 24 & 2 & -8 & 0 & 0 & 0 \\
\hline & -0.3289908 & 1.5 & 24 & 1 & -4 & 0 & 0 & 0 \\
\hline & -0.3287770 & 0.5 & 4 & 7 & -7 & 1 & 0 & 0 \\
\hline & * -0.3172244 & 2.5 & 12 & 7 & -7 & 1 & 0 & 0 \\
\hline & * -0.3171493 & 2.5 & 6 & 0 & 0 & 1 & -1 & 0 \\
\hline & * -0.3123477 & 2.5 & 6 & 0 & 0 & 1 & 1 & 0 \\
\hline & * -0.3119088 & 2.5 & 12 & 7 & -7 & 1 & 0 & 0 \\
\hline & * -0.2728708 & 3.5 & 8 & 0 & 0 & 1 & 1 & 0 \\
\hline & * -0.2728677 & 3.5 & 16 & 7 & -7 & 1 & 0 & 0 \\
\hline & * -0.2626233 & 3.5 & 8 & 0 & 0 & 1 & -1 & 0 \\
\hline & * -0.2624250 & 3.5 & 16 & 7 & -7 & 1 & 0 & 0 \\
\hline & * -0.2587919 & 3.5 & 16 & 7 & -7 & 1 & 0 & 0 \\
\hline 27 & * -0.3734808 & 0.5 & 4 & 9 & -9 & 1 & 0 & 1 \\
\hline
\end{tabular}




\begin{tabular}{|c|c|c|c|c|c|c|c|}
\hline * -0.3607827 & 0.5 & 12 & 6 & 12 & 0 & 0 & 1 \\
\hline * -0.3587615 & 1.5 & 4 & 0 & 0 & 1 & -1 & 1 \\
\hline * -0.3586044 & 1.5 & 8 & 9 & -9 & 1 & 0 & 1 \\
\hline * -0.3580457 & 1.5 & 4 & 0 & 0 & 1 & 1 & 1 \\
\hline * -0.3566086 & 0.5 & 12 & 3 & 6 & 0 & 0 & 1 \\
\hline-0.3522638 & 0.5 & 12 & 0 & 9 & 0 & 0 & 1 \\
\hline-0.3517280 & 0.5 & 12 & 0 & 9 & 0 & 0 & -1 \\
\hline-0.3500536 & 0.5 & 12 & 3 & -12 & 0 & 0 & 1 \\
\hline-0.3492247 & 0.5 & 12 & 6 & 12 & 0 & 0 & 1 \\
\hline-0.3482682 & 0.5 & 12 & 3 & -12 & 0 & 0 & 1 \\
\hline-0.3467737 & 0.5 & 12 & 3 & 6 & 0 & 0 & 1 \\
\hline-0.3466080 & 0.5 & 4 & 9 & -9 & 1 & 0 & 1 \\
\hline-0.3461131 & 0.5 & 12 & 3 & 6 & 0 & 0 & -1 \\
\hline-0.3452044 & 0.5 & 12 & 6 & 12 & 0 & 0 & 1 \\
\hline-0.3448586 & 0.5 & 12 & 6 & 12 & 0 & 0 & -1 \\
\hline * -0.3448234 & 1.5 & 24 & 3 & -12 & 0 & 0 & 1 \\
\hline * -0.3439540 & 1.5 & 24 & 3 & 6 & 0 & 0 & 1 \\
\hline * -0.3438163 & 1.5 & 24 & 6 & 12 & 0 & 0 & 1 \\
\hline-0.3436128 & 0.5 & 2 & 0 & 0 & 1 & 1 & 1 \\
\hline-0.3433155 & 0.5 & 12 & 3 & -12 & 0 & 0 & 1 \\
\hline-0.3432208 & 0.5 & 12 & 3 & -12 & 0 & 0 & -1 \\
\hline-0.3429195 & 0.5 & 12 & 0 & 9 & 0 & 0 & 1 \\
\hline-0.3422904 & 0.5 & 2 & 0 & 0 & 1 & -1 & 1 \\
\hline * -0.3359171 & 2.5 & 12 & 9 & -9 & 1 & 0 & 1 \\
\hline * -0.3358955 & 2.5 & 6 & 0 & 0 & 1 & 1 & 1 \\
\hline * -0.3337237 & 2.5 & 6 & 0 & 0 & 1 & -1 & 1 \\
\hline * -0.3336028 & 2.5 & 12 & 9 & -9 & 1 & 0 & 1 \\
\hline * -0.3072962 & 3.5 & 16 & 9 & -9 & 1 & 0 & 1 \\
\hline * -0.3072094 & 3.5 & 8 & 0 & 0 & 1 & -1 & 1 \\
\hline
\end{tabular}




\begin{tabular}{|c|c|c|c|c|c|c|c|c|}
\hline & * -0.3017642 & 3.5 & 8 & 0 & 0 & 1 & 1 & 1 \\
\hline & * -0.3016915 & 3.5 & 16 & 9 & -9 & 1 & 0 & 1 \\
\hline & * -0.3000848 & 3.5 & 16 & 9 & -9 & 1 & 0 & 1 \\
\hline & * -0.2734043 & 4.5 & 20 & 9 & -9 & 1 & 0 & 1 \\
\hline & * -0.2733890 & 4.5 & 10 & 0 & 0 & 1 & 1 & 1 \\
\hline & * -0.2632354 & 4.5 & 10 & 0 & 0 & 1 & -1 & 1 \\
\hline & * -0.2630937 & 4.5 & 20 & 9 & -9 & 1 & 0 & 1 \\
\hline & * -0.2593492 & 4.5 & 20 & 9 & -9 & 1 & 0 & 1 \\
\hline & * -0.2582988 & 4.5 & 10 & 0 & 0 & 1 & 1 & 1 \\
\hline & $*-0.2575865$ & 4.5 & 10 & 0 & 0 & 1 & -1 & 1 \\
\hline 36 & * -0.3735823 & 0 & 1 & 0 & 0 & 1 & 1 & 1 \\
\hline & * -0.3667362 & 1 & 2 & 0 & 0 & 1 & -1 & 1 \\
\hline & * -0.3555115 & 2 & 3 & 0 & 0 & 1 & 1 & 1 \\
\hline & -0.3517758 & 0 & 1 & 0 & 0 & 1 & 1 & 1 \\
\hline & -0.3485718 & 0 & 1 & 0 & 0 & 1 & 1 & 1 \\
\hline & -0.3430154 & 1 & 2 & 0 & 0 & 1 & -1 & 1 \\
\hline & -0.3400599 & 1 & 2 & 0 & 0 & 1 & -1 & 1 \\
\hline & * -0.3398439 & 3 & 4 & 0 & 0 & 1 & -1 & 1 \\
\hline & -0.3398327 & 2 & 3 & 0 & 0 & 1 & 1 & 1 \\
\hline & * -0.3386439 & 3 & 4 & 0 & 0 & 1 & 1 & 1 \\
\hline & * -0.3381808 & 3 & 4 & 0 & 0 & 1 & -1 & 1 \\
\hline & * -0.3204399 & 4 & 5 & 0 & 0 & 1 & 1 & 1 \\
\hline & * -0.3173265 & 4 & 5 & 0 & 0 & 1 & -1 & 1 \\
\hline & * -0.3162180 & 4 & 5 & 0 & 0 & 1 & 1 & 1 \\
\hline & * -0.2977774 & 5 & 6 & 0 & 0 & 1 & -1 & 1 \\
\hline & $*_{-}-0.2923236$ & 5 & 6 & 0 & 0 & 1 & 1 & 1 \\
\hline & * -0.2898917 & 5 & 6 & 0 & 0 & 1 & -1 & 1 \\
\hline & * -0.2721993 & 6 & 7 & 0 & 0 & 1 & 1 & 1 \\
\hline & * -0.2638050 & 6 & 7 & 0 & 0 & 1 & -1 & 1 \\
\hline
\end{tabular}




\begin{tabular}{|llllllll|}
$*_{-}-0.2594966$ & 6 & 7 & 0 & 0 & 1 & 1 & 1 \\
$*_{-}-0.2565594$ & 6 & 7 & 0 & 0 & 1 & -1 & 1 \\
$*-0.2563581$ & 6 & 7 & 0 & 0 & 1 & 1 & 1 \\
\hline \hline
\end{tabular}

TABLE I. Lowest energies, degeneracy and quantum numbers for the samples $N=9,12,21$, 27, 36. Components of vectors $k$ are in units of $2 \pi / N$. In the 3 last columns, 1 means invariant under the symmetry, 0 means no symmetry and -1 means a phase factor under the symmetry $(j$ for the rotation of $2 \pi / 3$ and -1 for the two others). Stars stand for the Quasi-Degenerate-Joint-States (QDJS).

\begin{tabular}{|cc|cc|}
\hline \hline $\mathbf{k}$ & $\mathcal{P}_{\mathbf{k}}$ & $\rho$ & multiplicity \\
\hline $\mathbf{k}=\mathbf{0}$ & $\mathcal{P}$ & $\mathcal{R}_{2 \pi / 3} \psi=\psi$ & 1 \\
& & $\mathcal{R}_{\pi} \psi= \pm \psi$ & 2 \\
& & $\mathcal{R}_{2 \pi / 3} \psi=j \psi$ & \\
& & $\mathcal{R}_{\pi} \psi= \pm \psi$ & 2 \\
\hline $\mathbf{k}= \pm \mathbf{k}_{0}$ & $<\mathcal{R}_{2 \pi / 3}>$ & $\mathcal{R}_{2 \pi / 3} \psi=\psi$ & 4 \\
& & $\mathcal{R}_{2 \pi / 3} \psi=j \psi$ & 3 \\
\hline $\mathbf{k}=-\mathbf{k} \neq \mathbf{0}$ & $\left\{I d, \mathcal{R}_{\pi}\right\}$ & $\mathcal{R}_{\pi} \psi= \pm \psi$ & 6 \\
\hline other $\mathbf{k}$ & $\{I d\}$ & & \multirow{2}{*}{} \\
\hline \hline
\end{tabular}

TABLE II. Irreducible Representations (IR) when there is no axial reflection $(l m \neq 0$ or $l \neq m)$.

First column: vector of the Brillouin zone. Second column: subgroup which keeps this vector invariant; $\left\langle\mathcal{R}_{2 \pi / 3}>\right.$ stands for the group generated by $\mathcal{R}_{2 \pi / 3}$. Third column: IR list of this subgroup, with the phase factor associated to the transformation; $\psi$ is a wavefunction. Fourth column: multiplicity of each eigenvalue found in this IR. 


\begin{tabular}{|c|c|c|c|}
\hline $\mathbf{k}$ & $\mathcal{P}_{\mathbf{k}}$ & $\rho$ & multiplicity \\
\hline \multirow[t]{2}{*}{$\mathbf{k}=\mathbf{0}$} & \multirow[t]{2}{*}{$\mathcal{P}$} & $\begin{aligned} \mathcal{R}_{2 \pi / 3} \psi & =\psi \\
\mathcal{R}_{\pi} \psi & = \pm \psi \\
\sigma_{x} \psi & = \pm \psi\end{aligned}$ & 1 \\
\hline & & $\begin{aligned} \mathcal{R}_{2 \pi / 3} \psi & =j \psi \\
\mathcal{R}_{\pi} \psi & = \pm \psi\end{aligned}$ & 2 \\
\hline \multirow[t]{2}{*}{$\mathbf{k}= \pm \mathbf{k}_{0}$} & \multirow[t]{2}{*}{$<\mathcal{R}_{2 \pi / 3}, \sigma_{x}>$} & $\begin{aligned} \mathcal{R}_{2 \pi / 3} \psi & =\psi \\
\sigma_{x} \psi & = \pm \psi\end{aligned}$ & 2 \\
\hline & & $\begin{array}{l}\mathcal{R}_{2 \pi / 3} \psi=j \psi \\
\sigma_{x} \mathcal{R}_{\pi} \psi= \pm \psi\end{array}$ & 4 \\
\hline $\mathbf{k}=-\mathbf{k} \neq \mathbf{0}$ & $<I d, \mathcal{R}_{\pi}, \sigma_{\mathbf{k}}>$ & $\begin{aligned} \mathcal{R}_{\pi} \psi & = \pm \psi \\
\sigma_{\mathbf{k}} \psi & = \pm \psi\end{aligned}$ & 3 \\
\hline$\sigma_{\mathbf{k}} \mathbf{k}=\mathbf{k} \neq \mathbf{0}$ & $\left\{I d, \sigma_{\mathbf{k}}\right\}$ & $\sigma_{\mathbf{k}} \psi= \pm \psi$ & 6 \\
\hline other $\mathbf{k}$ & $\{I d\}$ & & 12 \\
\hline
\end{tabular}

TABLE III. Idem as in Table II, when there is an axial reflection $(l m=0$ or $l=m)$. 


\begin{tabular}{|c|c|c|c|c|c|c|}
\hline & $I$ & $-I$ & $p_{3}$ & $-p_{3}$ & $p_{2}$ & $-p_{2}$ \\
\hline$N_{e l}$ & 1 & 1 & 2 & 2 & 3 & 3 \\
\hline$\Gamma_{0}$ & 1 & 1 & 1 & 1 & 1 & 1 \\
\hline$\Gamma_{1}$ & 1 & 1 & 1 & 1 & -1 & -1 \\
\hline$\Gamma_{2}$ & 2 & 2 & -1 & -1 & 0 & 0 \\
\hline$\Gamma_{0}^{\prime}$ & 1 & -1 & 1 & -1 & $i$ & $-i$ \\
\hline$\Gamma_{1}^{\prime}$ & 1 & -1 & 1 & -1 & $-i$ & $i$ \\
\hline$\Gamma_{2}^{\prime}$ & 2 & -2 & -1 & 1 & 0 & 0 \\
\hline$\phi$ & 0 & $2 \pi$ & $4 \pi / 3$ & $2 \pi / 3$ & $\pi$ & $\pi$ \\
\hline $\operatorname{Tr}^{(S)} \mathcal{R}(\phi)$ & $d$ & $(-1)^{2 S} d$ & $\frac{\sin \left(\frac{2 \pi}{3} d\right)}{\sin \left(\frac{2 \pi}{3}\right)}$ & $\frac{\sin \left(\frac{2 \pi}{3} d\right)}{\sin \left(\frac{2 \pi}{3}\right)}$ & $\sin \left(\frac{\pi}{2} d\right)$ & $\sin \left(\frac{\pi}{2} d\right)$ \\
\hline
\end{tabular}

TABLE IV. Character table for the spin-rotation group of $S U(2)$ involved in the Néel state; $p_{2}$ and $p_{3}$ denote the class of the spins rotations associated to 2 and 3 sublattice permutations. The number of elements in each class is $N_{e l}$. The rotation angle associated to each class is $\phi$ and the trace of the rotation in the subspace of fixed spin $S$ is given in the last line, where $d=2 S+1$. 


\section{FIGURES}

FIG. 1. The classical Néel ground-state: on each $i j k$ triangle $\mathbf{s}_{\mathbf{i}}+\mathbf{s}_{\mathbf{j}}+\mathbf{s}_{\mathbf{k}}=\mathbf{0}$. This defines three sublattices $A, B, C$ on which the spins are ferromagnetically aligned; the angle between the spins of two sublattices is $2 \pi / 3$. For a given planar upwards triangular plaquette described in the counter-clockwise direction, the spins can rotate clockwise or counter-clockwise, corresponding to the two different helicities: here a positive helicity is assumed.

FIG. 2. Energy spectra of Eq.11 versus $\left.\mathbf{S}^{2}=S(S+1) ; a\right)$ complete spectrum for $N=9$ and 12; b) lowest energies for $N=21$ and $N=27$. The horizontal and vertical scales have been enlarged by the same factor so that the slope of the energy per bond versus $\mathrm{S}(\mathrm{S}+1)$ can be compared. One sees on these graphs that this slope goes rapidly to zero. The straight line is a guide for the eye to link the low lying energy levels called QDJS (for quasi-degenerate joint states).

FIG. 3. Moment of inertia versus sample size

FIG. 4. a) Enlargement of the low energy levels for $N=9$ versus $S(S+1)$. Three-legs-symbols family and - family are essential ingredients of an à la Néel symmetry breaking. They respectively span the subspace of quasi-classical Néel ground-states and the subspace of the long wave-length $\mathbf{k} \neq \mathbf{0}$ excitations: i.e. the magnons. Black triangles represent the chiral states. Open triangles are states invariant under rotation of $2 \pi / 3$ and odd under axial symmetry. $b$ ) same as in $a$ ) for 27 spins. c) same as $b)$ with $\frac{S(S+1)}{6 N I_{\perp}}$ subtracted from the whole spectrum.

FIG. 5. Schematic of the free dynamics of a symmetric top

FIG. 6. Comparison between the exact spectrum and a fitted symmetric top for the $N=27$ sample : 1, 2 and 3 stand for the $\Gamma_{1}, \Gamma_{2}, \Gamma_{3}$ IRs (see text); $\square$ : spectrum of an ideal symmetric top

(the isotropic term $\frac{S(S+1)}{6 N I_{\perp}}$ is subtracted from the spectrum to focus the comparison on the second term of Eq.12); For $N=27$, all levels of the ideal top ( $\square$ ) are doubly degenerate (we do not take into account the trivial $2 S+1$ magnetic degeneracy). In the exact spectrum, the levels 1 and 2 are simply degenerate and the 3 levels are doubly degenerate. Perfect agreement between the two spectra would necessitate a quasi degeneracy of 1 and 2 levels. 
FIG. 7. Spin susceptibilities of the THA on finite samples. Fig.7a: $\chi_{\perp}$ (triangles) and $\chi_{\|}$ (square) normalized by its classical value for the $N=3 p$-samples; large symbols stand for results obtained from the QDJS analysis; small symbols show the finite-size results of spin-wave calculation of Chubukov et al [45]. Points represent spin-wave results and indicate the infinite size extrapolation. Fig.7b: $\chi_{\|}$of the $N=3 p+1$-samples; symbols are the same as in $a$ ).

FIG. 8. The $N=13$ sample and the tiling of the infinite lattice. Numbers stand for the spin. Prime (resp. double prime) means that the original spin is rotated by $\pm 2 \pi / 3$ (resp. $\pm 4 \pi / 3$ ).

FIG. 9. Opposite of the ground-state energy of the $N=21$ and $N=19$ samples as a function of the spin-rotation angles $\phi, \psi$ attached to the translations $\mathbf{u}_{1}, \mathbf{u}_{2}$. The spectrum depends only on the angles $\Phi=l \phi+m \psi, \Psi=-m \phi+(l+m) \psi$ attached to the translations $\mathbf{T}_{1}$, $\mathbf{T}_{2}$. In the $N=3 p$ case, the absolute minimum of the energy is obtained for angles $\Phi, \Psi$ equal to 0 or $\pm \frac{2 \pi}{3}$ characterizing the classical Néel states and only $(\Phi, \Psi)=\left( \pm \frac{2 \pi}{3}, \mp \frac{2 \pi}{3}\right)$ in the $N=3 p+1$ samples.

FIG. 10. Spectra of $N=3 p+1$ samples versus $S_{3}^{2}$. Note the tower of states, only doubly degenerate, and its collapse to the absolute ground-state with increasing $N$.

FIG. 11. Finite-size scaling of the ground-state energy per bond and comparison with spin-wave results. a) three-legs symbols: diagonalization results for the $N=3 p$-samples; black triangles: averaged values computed thanks to Eq.18; small open triangles: spin-wave results; dotted line: $N^{-3 / 2}$ fit on large $N$ spin-wave results. $b$ ) same as in $a$ ) except crosses: diagonalization results for the $N=3 p+1$-samples. $c$ ) averaged values of $\left\langle 2 \mathbf{s}_{i} \cdot \mathbf{s}_{j}\right\rangle$ versus $N^{-3 / 2}$; dotted line $: N^{-3 / 2}$ fit to these results of diagonalizations (see text).

FIG. 12. Static structure function in the ground-state of the $N=3 p$ samples versus the $\mathbf{k}$-vector modulus.

FIG. 13. Néel order parameter as a function of the sample size. The order parameter is normalized by its maximum value (see Eq.25 and Eq.26). Triangles (resp. squares) stand for $N=3 p$ (resp. $N=3 p+1$ )-samples; black symbols stand for diagonalization results; open symbols stand for first order spin-wave results $\left(2<M_{z}>\right.$ of Eq.28); dotted lines : large $N$ fits $(N>5000)$ of first-order spin-wave results $\left(X_{N}=X_{\infty}+a N^{-1 / 2}\right)$. 